\title{
Expanded Indications for Transcervical Thymectomy in the Management of Anterior Mediastinal Masses
}

\author{
Maher E. Deeb, MD, Clay J. Brinster, BSc, John Kucharzuk, MD, Joseph B. Shrager, MD, \\ and Larry R. Kaiser, MD
}

Section of General Thoracic Surgery, Division of Cardiothoracic Surgery, Department of Surgery, University of Pennsylvania School of Medicine, Philadelphia, Pennsylvania

Background. Transcervical thymectomy (TCT) is an accepted though controversial approach for thymectomy in myasthenia gravis (MG). The suggestion of thymoma on computed tomography (CT) has been considered a contraindication to TCT. We sought to determine whether the indications for TCT could be safely expanded to include selected patients with thymomas as well as other types of anterior mediastinal masses.

Methods. Between January 1992 and September 1999, we performed 121 TCTs: 98 in patients with MG and 23 in patients without MG. The patients' records were retrospectively reviewed.

Results. Among the 98 MG patients, 28 had CT scans suspicious for thymoma. Of these, 14 had a thymoma pathologically. These were classified as stage I (5), stage II (8), and stage III (1). Five patients required extension of the incision for completion of the procedure. There have

$S$ ince it was first described by Schumacher and Roth in 1912 [1], transcervical thymectomy (TCT) has been employed mainly for patients with myasthenia gravis (MG) without thymoma. The use of the procedure beyond this indication has been reported in only a handful of cases [2-4]. The existence of a thymoma has generally been considered a contraindication to the transcervical approach and a mandate for a median sternotomy. In 1988, TCT was modified and improved by the introduction of the Cooper retractor [5]. This instrument significantly improved exposure of the anterior mediastinum and facilitated safe and extensive exploration of this area by TCT.

Since 1992, the transcervical approach has been our approach of choice in all cases of thymectomy for myasthenia gravis without thymoma. We have been so satisfied with the exposure of the anterior mediastinum during these cases that as we gained experience with TCT, we gradually extended its indications to include three other major groups: group 1, myasthenic and nonmyasthenic patients with preoperative suspicion of thymoma less than $5 \mathrm{~cm}$ in diameter without invasion of

Accepted for publication March 27, 2001.

Address reprint requests to Dr Kaiser, Hospital of the University of Pennsylvania, 6th Floor, Silverstein Building, 3400 Spruce St, Philadelphia, PA 19104; e-mail: kaiser@mail.med.upenn.edu. been no thymoma recurrences to date with a mean follow-up of 48 months (range 3 to 96 months). In the 23 patients without MG, 12 had new anterior mediastinal masses, 4 had a history of treated lymphoma, 1 had a history of treated germ cell tumor, and 6 had suspected mediastinal parathyroid adenoma. Diagnostic tissue was obtained in all patients undergoing the procedure for diagnosis, and in 4 of 6 patients, a parathyroid adenoma was successfully resected.

Conclusions. Transcervical exploration and thymectomy offers a less invasive approach to the diagnosis and/or definitive treatment of selected anterior mediastinal masses. We suggest that it is appropriate to expand its use to several clinical scenarios beyond the typical indication of thymectomy in MG patients without thymoma.

(Ann Thorac Surg 2001;72:208-11)

(C) 2001 by The Society of Thoracic Surgeons

surrounding structures by computed tomography (CT) scan; group 2, selected nonmyasthenic patients with anterior mediastinal masses requiring diagnosis; and group 3, patients with suspicion of mediastinal or intrathymic parathyroid adenoma.

In this report, we review our experience when TCT was performed for these three groups of patients.

\section{Material and Methods}

Between February 1992 and September 1999, we performed 121 TCTs: 98 patients had MG and 23 did not. The operations were performed essentially as described by Cooper and associates [5]. The neck incision was approximately $10 \mathrm{~cm}$ in length. Total thymectomy was the intended procedure for both MG and thymoma. The inpatient and outpatient medical records, operative notes, and pathology reports were reviewed. Additional follow-up was obtained by calling the patients, their primary physician, or their neurologist.

\section{Results}

There were no perioperative mortalities. One late death occurred 4 months postoperatively due to septic shock 
and unexplained erythroderma. The mean operative time was 95 minutes (range 40 to 120 minutes). There were five cases $(13 \%)$ with morbidity: one seroma that required percutaneous drainage, one wound infection that required reexploration for drainage, one case of transient hypocalcemia, one cerebrovascular accident, and one case of atrial fibrillation. The latter two complications occurred in patients who required extension to a median sternotomy for complete resection, while the first three occurred in patients who did not require extension of the procedure beyond the neck incision. These three complications did require readmission to the hospital. All procedures that did not require extension were performed on an outpatient basis except those in which hypocalcemia was deemed to be a risk postoperatively.

\section{Group 1: Suspicion of Thymoma}

Twenty-eight patients from the entire group of 121 patients had a CT scan suspicious for thymoma. Fourteen of these 28 patients had pathology-proven thymoma, and all of these patients were myasthenic. Five patients had thymoma stage I, 8 stage II, and 1 stage III. Five patients in this group required extension of the procedure beyond the transcervical approach in order to have complete resection: 3 patients had median sternotomy, 1 had an upper sternal split, and 1 had left video-assisted thoracoscopy (VATS). The reasons for conversion were 1 patient with stage I thymoma who had undergone previous resection needed extension due to extensive adhesions; and 3 patients with stage II thymoma required extension due to proximity to the phrenic nerve in the first, adhesions in the second, and difficulty due to the size of the tumor in the third. The patient with stage III thymoma was converted to median sternotomy due to invasion of the pericardium and right upper lobe.

Mean follow-up for this group was 48 months (range 3 to 96 months). One patient was lost to follow-up. No recurrences have occurred. Postoperative radiation was given to all patients beyond stage I except 2 patients in stage II. One of these had only microinvasion of the capsule and the second had already been maximally radiated due to thymic irradiation as a child and additional irradiation as an adult for breast cancer.

\section{Group 2: Anterior Mediastinal Mass}

Seventeen nonmyasthenic patients had anterior mediastinal masses by CT scan. (5 were suspicious for thymoma and are included in group 1 as well). In 12 patients, the mass was discovered during evaluation of unrelated disease. Four patients had a history of previously treated lymphoma and 1 had a history of germ cell tumor. The pathology of the latter demonstrated only follicular hyperplasia, and there has been no later evidence of recurrent germ cell tumor. In 2 patients with previous Hodgkin's lymphoma, recurrence was diagnosed in both. The other 2 patients with previous lymphoma were found to have follicular hyperplasia and normal thymus, respectively, and there has been no later evidence of recurrent lymphoma in these patients.

Of the remaining 12 patients without a history of

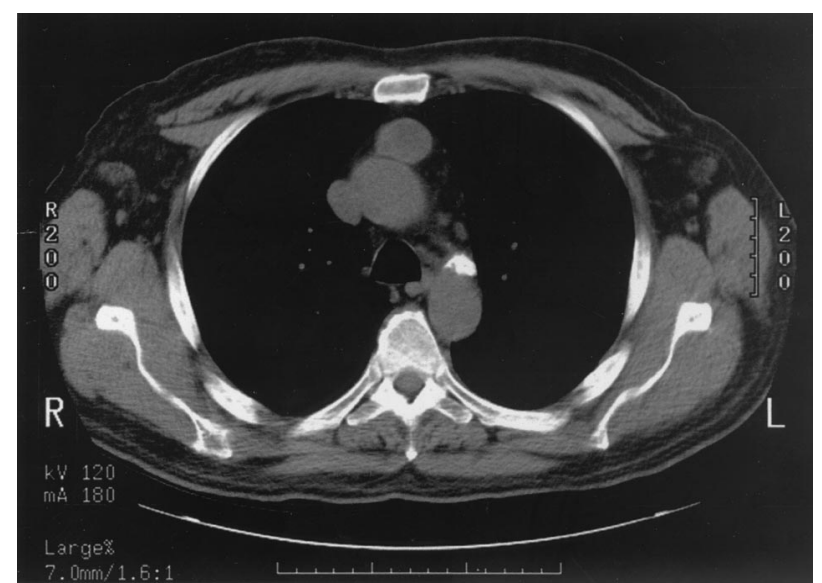

Fig 1. A well-circumscribed mass $(3 \times 2 \mathrm{~cm})$ in the anterior mediastinum that was completely resected through the transcervical approach. Although the final pathology in this case was nonkeratizing carcinoma, this scan is fairly typical of the larger thymomas successfully approached transcervically in this series.

malignancy, 11 patients had benign pathology: thymic cyst (3), follicular hyperplasia (6), right upper lobe hamartoma (1) (following negative right VATS), and normal thymus (1). One patient was found to have nonkeratizing carcinoma of the thymus gland. This patient was a 58-year-old male who had undergone renal transplantation 4 years previously. A 3-cm anterior mediastinal mass was found on CT during the evaluation of severe erythroderma. The mass was well encapsulated and easily removed through the transcervical approach (Fig 1). The patient died 4 months after surgery due to septic shock, and autopsy showed no residual tumor.

Only 1 patient in this group needed extension of the TCT to partial sternotomy. This was a 50 -year-old female with a suspicious mass, which appeared to be in the anterior mediastinum, discovered during evaluation for cough. She had undergone a previous negative right VATS at an outside hospital. During TCT, no anterior mass was found, but rather a right upper lobe lesion was identified and this was resected by wedge performed through a partial upper sternal split. The final pathology was hamartoma.

\section{Group 3: Parathyroid Adenoma}

Six patients were explored via TCT for suspected mediastinal parathyroid adenoma. These patients are described in Table 1. In 2 patients (patients nos. 5 and 6), mediastinal exploration was performed during the same anesthesic after negative neck exploration in 1 and reexploration in the other. In both of these patients, we failed to demonstrate intrathymic adenoma. In 1 patient (no. 4), TCT was the primary procedure as a preoperative sestamibi scan showed a parathyroid adenoma to be in the mediastinum. Three patients (nos. 1 to 3 ) had a previous negative neck exploration and later sestamibi scan showing mediastinal adenoma. Two of these patients had a failed trial of embolization before they were referred to us. Intrathymic parathyroid adenoma was found in each of these patients and they were all cured. 
Table 1. Clinical Characteristics of the 6 Patients Who Underwent TCT for Suspected Mediastinal Parathyroid Adenoma

\begin{tabular}{|c|c|c|c|c|c|}
\hline & Age/Gender & Neck Exploration & Normal PT Glands & Localization Studies & Adenoma Location \\
\hline 1 & $55 / \mathrm{F}$ & Previous & 4 & CT (+) Sestamibi $(-)$ & Intrathymic \\
\hline 2 & $30 / \mathrm{F}$ & Previous & $\begin{array}{l}3 \text { Rt Sup gland not } \\
\text { found }\end{array}$ & $\begin{array}{l}\text { MRI (+) Sestamibi }(+) \mathrm{s} / \mathrm{p} \\
\text { embolization }\end{array}$ & Intrathymic \\
\hline 3 & $18 / \mathrm{F}$ & Previous & 4 & $\begin{array}{l}\text { MRI (+) Sestamibi }(+) \mathrm{s} / \mathrm{p} \\
\text { embolization }\end{array}$ & Intrathymic \\
\hline 4 & $50 / \mathrm{M}$ & Mediastinal exploration only & 0 & CT $(+)$ Sestamibi $(+)$ & Intrathymic \\
\hline 5 & $46 / \mathrm{F}$ & $\begin{array}{l}\text { Concomitant first } \\
\text { exploration }\end{array}$ & $\begin{array}{l}2 \text { Rt Sup and Lt } \\
\text { Inf not found }\end{array}$ & $\begin{array}{l}\text { Intra-op US No pre op localization } \\
\text { studies }\end{array}$ & Rt Thyroid lobe \\
\hline 6 & $73 / \mathrm{M}$ & Concomitant Re-exploration & 4 & $\begin{array}{l}\text { CT \& MRI (-) Angio (-) Venous } \\
\text { sampling (-) }\end{array}$ & No adenoma found \\
\hline
\end{tabular}

MRI = magnetic resonance imaging; $\mathrm{s} / \mathrm{p}=$ status post.

\section{Comment}

Nearly all of the available literature on TCT addresses the utilization of this procedure in patients with MG without thymoma. There are few reports that consider TCT for lesions suspicious for thymoma. Kark and Kirschner [3] reported in 1971 on the use of TCT for 7 patients with high-lying thymoma, but no substantial follow-up was reported. The largest experience reported is from the Mount Sinai group [4] that consists of 46 patients with thymoma resected by TCT alone and another 18 patients in whom a complementary mediastinotomy was required for complete resection. In most of the patients in this series (from early in the era of CT), the thymomas were not suspected preoperatively, and an improved survival was noted in occult thymomas operated upon by TCT versus preoperatively identified thymomas operated upon by sternotomy. The fact that, in our series, $83 \%$ of patients with suspected thymoma had their resection completed transcervically, and that in those with verified thymoma there have been no recurrences with a mean follow-up of 48 months, indicates a role for TCT in these patients. Although thymoma recurrence can certainly occur beyond this follow-up period, there would be no reason to expect a high rate of late recurrence as none of our stage I or II patients had positive resection margins.

The issue that begs addressing is which patients with suspected thymoma should be considered candidates for TCT. Certainly, those with evidence of frank invasion of surrounding structures (stage III) should not be approached transcervically. Even using these criteria, however, Kark and Kirschner's [3] and Papatestas and associates' [4] experience, as well as our own, is that extension of the procedure will be required in some cases. Although in 1 patient in our thymoma group extension was required by tumor size alone $(5 \mathrm{~cm})$, we did achieve complete excision in another patient with a 5 -cm thymoma. In no lesion $4 \mathrm{~cm}$ or less in diameter was conversion required strictly on the basis of the size of the tumor. Based upon this experience, we feel that $4 \mathrm{~cm}$ is the appropriate upper limit for attempting the transcervical approach. The limited space between the sternum anteriorly and the innominate vein and mediastinum posteriorly renders safe dissection and delivery of tumors greater than $4 \mathrm{~cm}$ in diameter problematic. Even using this size limit, there will be occasional conversions to larger incisions due to adhesions or unexpected invasion of surrounding structures. If at the time of TCT there is insufficient visualization of the anterior mediastinum and the phrenic nerves, or if there are not clear planes of dissection, then extension of the procedure should be performed without hesitation. The relatively high conversion rate in the pathologically proven thymoma cases in this series $(36 \%)$ reflects the fact that these criteria evolved with our experience, and the fact that we do not hesitate to extend the incision to a partial or full sternotomy if the safety or completeness of the operation seem to be in any way compromised. It should be emphasized that although $36 \%$ of patients with suspected thymoma required an extension of the procedure, conversely, 64\% of patients with small thymomas were successfully and, we think, adequately treated with a minimally morbid, outpatient procedure instead of a median sternotomy.

The transcervical approach to the anterior mediastinum can also be helpful for diagnostic procedures evaluating other types of masses in much the same way that Chamberlain procedure can be employed but with greater versatility. With advances in imaging techniques, the incidental small anterior mediastinal mass has become quite common. Furthermore, it is not unusual for these lesions to be out of reach of both mediastinoscopy and anterior mediastinotomy. Typically, such lesions are located directly beneath the sternum or slightly to the right in the anterior superior mediastinum. In 2 patients out of 5 with previous malignancy, we documented recurrence with TCT, while the other 3 patients were found to have follicular hyperplasia, and none of these patients were later found to have a missed recurrence. Eleven of our 12 nonmyasthenic patients with anterior mediastinal masses and no history of malignancy had benign pathology, while only 1 had carcinoma. Without the transcervical approach, these patients would likely have undergone median sternotomy for their benign diseases.

Parathyroid adenoma is a common problem. Eightyfive percent of patients with primary hyperparathyroid- 
ism have solitary parathyroid adenoma [6]. Most of the adenomas are located in the neck, where surgical excision via neck exploration can be accomplished in up to $97 \%$ of the patients without significant morbidity [7]. For this reason, most endocrine surgeons do not perform localization studies before neck exploration, and these studies are typically performed only after failed neck exploration.

Mediastinal parathyroid adenoma was first described by Churchill in 1932 [8]. The thymus gland is the most common ectopic site for parathyroid adenoma and constituted $17 \%$ of 222 patients with missed single parathyroid adenoma who were operated upon at the National Institutes of Health [9]. Most surgeons utilize median sternotomy for mediastinal exploration for suspected parathyroid adenoma [9-11], but this approach is associated with a high morbidity rate. Russell and associates [10] reported a $21 \%$ incidence of chest complications after median sternotomy. Mediastinotomy has been reported by Schlinkert and associates [12] for anterior mediastinal exploration in cases of suspected mediastinal adenoma. TCT has been previously reported in few cases, including Wells' and Cooper's [2] report of 2 patients and Kark and Kirschner's [3] 1 patient.

Our experience with TCT for suspected mediastinal parathyroid adenoma included two failures in the 2 patients with no or negative preoperative localization studies, and four successes in the 4 patients with at least one positive preoperative localization study. The two failures occurred in patients who had two and four normal parathyroid glands identified in the neck, respectively. Thus, we can agree with Cooper's suggestion that in cases of negative exploration and three normal parathyroid glands, the mediastinum might be explored immediately by TCT if there is a surgeon available who is familiar with the technique. However, it is essential that three normal glands have been previously identified. In all other cases, a preoperative localization study would appear to dramatically increase the chance of successful exploration by TCT.

Finally, it is worth noting that some of the masses described herein may also have been approached by a video thoracoscopic technique. Thymectomy by VATS was reported by Mack and associates [13], and recently VATS was reported in resection of mediastinal parathyroid adenoma [14]. It is our feeling that the transcervical approach is less morbid than a VATS approach, particularly with regard to the absence of intercostal neuralgia after TCT, and for this reason, we favor the transcervical operation in situations where either approach is likely to be successful.

In summary, we report that the transcervical approach employed for TCT with the Cooper retractor can be successfully extended to three groups: (1) patients with suspected noninvasive thymoma less than $4 \mathrm{~cm}$ in size; (2) patients with simple anterior mediastinal masses with or without a previous history of mediastinal malignancy; and (3) patients with suspected mediastinal parathyroid adenoma. In the latter two groups, we have found that this approach can be used for lesions not reachable by the Chamberlain procedure and that might therefore otherwise require a sternotomy.

\section{References}

1. Schumacher ED, Roth J. Thymektomie bei cenem Fall von Morbus Basedowii mit Myasthenie, Mit a. d. Grezgeb d. Med Chir 1912;25:746.

2. Wells SA, Cooper JD. Closed mediastinal exploration in patients with persistent hyperparathyroidism. Ann Surg 1991;214:555-61.

3. Kark AE, Kirschner PA. Total thymectomy by the transcervical approach. Br J Surg 1971;58:323-6.

4. Papatestas AE, Pozner J, Genkins G, Kornfeld P, Matta RJ. Prognosis in occult thymoma in myasthenia gravis following transcervical thymectomy. Arch Surg 1987;122:1352-6.

5. Cooper JD, Al-Jilaihawa AN, Pearson FG, Humphrey JG, Humphrey HE. An improved technique to facilitate transcervical thymectomy for myasthenia gravis. Ann Thor Surg 1988;45:242-7.

6. Thompson NW, Eckhauser F, Harness J. The anatomy of primary hyperparathyroidism. Surgery 1982;92:814-21.

7. ReMine SG. Management of recurrent or persistent hyperparathyroidism. Probl Gen Surg 1985;2:440-9.

8. Bauer W, Freedman DD. Hyperparathyroidism epitomized: the case of Captain Charles E. Martell. Metabolism 1962;11: 21-9.

9. Jaskowiak N, Norton JA, Alexander HR, et al. A prospective trial evaluating a standard approach to reoperation for missed parathyroid adenoma. Ann Surg 1996;224:308-22.

10. Russell CF, Edis AJ, Scholz DA, Sheedy PF, Van Heerden JA. Mediastinal parathyroid tumors: experience with 38 tumors requiring median sternotomy for removal. Ann Surg 1981; 193:805-9.

11. Downey NJ. Meguigan A, Dolan SJ, Russell CF. Median sternotomy for parathyroid adenoma. Ir J Med Sc 1999;168: 13-6.

12. Schlinkert RT, Whitaker MD, Argueta R. Resection of selected mediastinal adenomas through an anterior medistinotomy. Mayo Clin Proc 1991;66:1110-3.

13. Mack MJ, Landreneau AP, Yim AP, Scruggs GR. Results of video-assisted thymectomy in patients with myasthenia gravis. J Thorac Cardiovasc Surg 1996;112:1352-9.

14. Medrano C, Hazelrigg RS, Landreneau JR, Boley MT, Shawgo T, Grasch A. Thoracoscopic resection of ectopic parathyroid glands. Ann Thorac Surg 2000;69:221-3. 